\title{
WEAK DENSITY OF THE FUNDAMENTAL GROUP SCHEME
}

\author{
HÉLÈNE ESNAULT AND VIKRAM B. MEHTA
}

\begin{abstract}
Let $X$ be a non-singular projective variety over an algebraically closed field $k$ of characteristic 0 . If $\pi_{1}^{\text {et }}(X)=0$, then for any ample line bundle $H$ on $X$, any semistable bundle $E$ on $X$ with all Chern classes 0 is trivial. Over $\mathbb{C}$, this a consequence of the fact that $\pi_{1}^{\text {top }}\left(X_{\text {an }}\right)$ is a finitely generated group and hence any representation into a linear group is residually finite. We prove an analog of this theorem in characteristic $p>0$. Semistable bundles with vanishing Chern classes are replaced by Nori semistable bundles, that is those which are semistable of degree 0 on any curve mapping to $X$. The étale fundamental group is replaced by Nori's fundamental group scheme $\pi^{N}(X)$ (14), which is the profinite completion of the tensor automorphism group scheme $\pi^{S}(X)$ of Nori semistable bundles ([9]) studied by Langer ([8] [9]).
\end{abstract}

\section{INTRODUCTION}

Let $X$ be a non-singular projective variety over the field $k=\mathbb{C}$ of complex numbers. Let $H$ be a very ample line bundle on $X$. Assume that $E$ is a stable vector bundle on $X$, with respect to $H$, with all the Chern classes $0=c_{i}(E) \in$ $H^{2 i}(X, \mathbb{Q}(i)), 1 \leq i \leq d=$ dimension $X$. Then it is classical [13] that $E$ carries an integrable connection with unitary underlying monodromy. If $E$ is assumed to be merely semistable, then one shows that there is a Jordan-Hölder filtration $0 \subset E_{0} \subset \ldots \subset E_{n}=E$ such that each $E_{i} / E_{i-1}$ is a stable bundle with all Chern classes 0 .

Assume now that one is working over $k$, an arbitrary algebraically closed field of characteristic 0 . If $X$ and $H$ are as above, and $E$ is a semistable bundle on $X$, with all Chern classes in $\ell$-adic cohomology $H^{2 i}\left(X, \mathbb{Q}_{\ell}(i)\right)$ trivial, then again it follows from the Lefschetz principle, that there is a filtration $0 \subset E_{0} \subset \ldots \subset E_{n}=E$, where each $E_{i} / E_{i-1}$ is a stable bundle with all Chern classes 0 .

For a stable vector bundle $E$ on $X$ with all Chern classes 0 over $k=\mathbb{C}$, let $\rho: \pi^{\text {top }}\left(X_{\text {an }}\right) \rightarrow U(r)$ be the associated irreducible unitary representation. Then the image $G$ of $\rho$ is a finitely generated subgroup of $G L(r, \mathbb{C})$. By Malcev theorem [10, $G$ is residually finite, that is $G$ injects into its profinite completion $\widehat{G}$. If $G \neq$ $\{1\}$, there is a homomorphism of $G$ onto a finite group, thus a homomorphism

Date: November 09, 2009.

1991 Mathematics Subject Classification. 13D40.

The first author is supported by the DFG Leibniz Preis, the SFB/TR45 and the ERC Advanced Grant 226257. 
from $\pi^{\text {top }}(X)$ onto a finite group. If one now assumes that $\pi^{\text {et }}(X)=0$, this implies that $G=\{1\}$, thus $E$ is trivial. If $E$ is only semistable, then the above argument implies that the associated graded bundle $\oplus_{i}\left(E_{i} / E_{i-1}\right)$ is trivial. On the other hand, $\pi^{\text {et }}(X)=0$ implies that $H_{\text {et }}^{1}(X, \mathbb{Z} / n)=\operatorname{Hom}\left(\pi_{1}^{\text {et }}(X), \mathbb{Z} / n\right)=0$, thus $\ell$-adic cohomology $H_{\text {et }}^{1}\left(X, \mathbb{Z}_{\ell}\right)$ is trivial, thus, by the comparison theorem, Betti cohomology $H^{1}\left(X_{\text {an }}, \mathbb{Z}\right)$ is trivial, thus by Hodge theory, $H^{1}\left(X, \mathcal{O}_{X}\right)=0$. So $E$, which is a successive extension of $\mathcal{O}_{X}$ by itself, is trivial as well.

Note that in characteristic 0 , any semistable $E$ on a smooth projective variety $X$ with all Chern classes 0 has the following property : If $C$ is a smooth curve and $f: C \rightarrow X$ is any map, then $f^{*} E$ is semistable of degree 0 on $C$. If $k=\mathbb{C}$, then $f^{*} E$ is thus filtered such that the graded bundle is a sum of bundles, each of which carries a connection with underlying unitary monodromy. This motivates the following:

Definition 1.1. Let $X$ be a projective variety over any algebraically closed field. Let $E$ be a vector bundle on $X$. Then $E$ is Nori semistable if for all smooth projective curves $C$, all morphisms $f: C \rightarrow X$, the bundle $f^{*}(E)$ is semistable on $C$, of degree 0 .

This notion has been introduced by Nori [14, Definition p.81]. We have seen above that in characteristic 0 , any semistable $E$ on a smooth $X$ with all Chern classes 0 is Nori semistable. It is indeed true for $k=\mathbb{C}$ and thus true for all $k=\bar{k}$ of characteristic 0 by the Lefschetz principle.

In characteristic $p>0$, a bundle can be stable and not Nori semistable, as Frobenius pull backs of semistable bundles are no longer semistable, even for curves [4, Theorem 1]. This is one reason why one works with Nori semistable bundles.

Let $\mathrm{Ns}(X)$ be the category of Nori semistable bundles on a smooth projective connected variety $X$ defined over an algebraically closed field $k$ of characteristic $p>0$. Nori shows [14, Lemma 3.6] that this is a $k$-linear, abelian, rigid tensor category. Fix a $k$-rational point $x$ in $X$. Let $\omega_{x}: \mathrm{Ns}(X) \rightarrow \mathrm{Vec}_{k}$ be the tensor functor $\left.W \rightarrow W\right|_{x}$. Then $\left(\operatorname{Ns}(X), \omega_{x}\right)$ is a Tannaka category, and one defines $\pi^{S}(X, x):=\operatorname{Aut}^{\otimes}\left(\operatorname{Ns}(X), \omega_{x}\right)$ to be the associated Tannaka group scheme.

This notation $\pi^{S}(X, x)$ was introduced in [1] on curves and [8] in any dimension. More precisely, in [9, 1.2], Langer mentions that $\mathrm{Ns}(X)$ is identical to the category of vector bundles $E$ which are numerically trivial, which means that both the tautological line bundle $\mathcal{O}(1)$ on $\mathbb{P}(E)$ and the one on $\mathbb{P}\left(E^{\vee}\right)$ are numerically effective. We recall this fact in Lemma 2.2. He also shows [8, Proposition 5.1] that $\mathrm{Ns}(X)$ is the category of strongly semistable reflexive sheaves with the property that $\operatorname{deg}\left(\operatorname{ch}_{1}(E) \cdot H^{d-1}\right)=\operatorname{deg}\left(\operatorname{ch}_{2}(E) \cdot H^{d-2}\right)=0$ for a fixed polarization $H$. So in particular, it does not depend on the chosen polarization.

Nori [14, Definition p.82] constructed the category $\mathcal{C}^{N}(X)$ of essentially finite bundles as the full subcategory of $\mathrm{Ns}(X)$ spanned by finite bundles, where finiteness 
is in the sense of Weil: $E$ is finite if there are two polynomials $f \neq g, f, g \in \mathbb{N}[T]$, such that $f(E) \cong g(E)$. Thus by definition,

$$
\pi^{S}(X, x) \rightarrow \pi^{N}(X, x)
$$

is the profinite completion homomorphism of the $k$-progroup scheme $\pi^{S}(X, x)$. We prove:

Theorem 1.2. Let $X$ be a smooth projective connected variety over an algebraically closed field of characteristic $p>0$. Let $x \in X(k)$. If $\pi^{N}(X, x)=\{1\}$, then $\pi^{S}(X, x)=\{1\}$ as well.

In characteristic 0 , one has $\pi^{N}(X, x)(k)=\pi^{\text {et }}(X, x)$, and thus Theorem 1.2 is a generalization of the theorem discussed above. Note also that if $k=\overline{\mathbb{F}}_{p}$, then every $E$ in $\operatorname{Ns}(X)$ is actually essentially finite, thus in this case $\pi^{S}(X, x)=\pi^{N}(X, x)$ and the theorem is trivial.

The main point of our article is to prove Theorem 1.2 over an arbitrary field of characteristic $p>0$. The proof is a variant of the proof of [3, Theorem 1.1]. Apart from the existence of quasi-projective moduli spaces due to Langer [7, Theorem 4.1], the main tool is Hrushovski's fundamental theorem [5, Corollary 1.2]. The difference with the proof of the main theorem in [3] relies in the choice of the sublocus of the moduli on which we ultimately wish to apply Hrushovski's theorem. In [3], we defined Verschiebung divisible subschemes of $M$ (see [3], Definition 3.6]), while here the notion which works is reversed. We do not discuss this in the note, but the locus we defined is rather Verschiebung multiplicative, that is if one moduli point $[E]$ lies in it, then $\left[F^{*} E\right]$ lies in it as well. Another difference is that in [3], even over $\overline{\mathbb{F}}_{p}$, we had to appeal to Hrushovski's theorem. In the present situation, where we deal with bundles going up by Frobenius, over $\overline{\mathbb{F}}_{p}$, we can appeal to Lange-Stuhler theorem [6, Satz 1.4]. Only for an arbitrary algebraically closed field $k$, does one use Hrushovski's theorem.

Acknowledgements: We would like to thank Holger Brenner, Laurent Ducrohet and Yves Laszlo for several helpful conversations. The second author would like to thank the SRB/TR45 at the University Duisburg-Essen for hospitality on several occasions during the last years.

\section{Proof of Theorem 1.2}

Throughout this section, $X$ is a smooth connected projective variety of dimension $d$ over an algebraically closed field $k$ of characteristic $p>0, F: X \rightarrow X$ is the absolute Frobenius morphism of $X, x \in X(k)$ is a rational point, and $H$ is a fixed ample line bundle on it. We prove Theorem 1.2 in a series of lemmas.

Lemma 2.1. If $\pi^{N}(X, x)=0$, then $\pi^{\mathrm{et}}(X, x)=0$.

Proof. As well known (see e.g. [2, Remarks 2.10]), thinking of $\pi^{\text {et }}(X, s)$ as a constant $k=\bar{k}$-progroup scheme, the $k$-homomorphism $\pi^{N}(X, x) \rightarrow \pi^{\text {et }}(X, x)$ is surjective. In fact, this is the pro-smooth quotient of $\pi^{N}(X, x)$. 
Recall [8, 1.2] that a bundle $E$ is said to be numerically effective if for any smooth projective curve $f: C \rightarrow X$, the minimal slope of $f^{*} E$ is nonnegative. This is equivalent to saying that the tautological line bundle $\mathcal{O}(1)$ on $\mathbb{P}(E)$ is numerically effective.

Lemma 2.2. (See [9, 1.2]) A vector bundle $E$ on $X$ is in $\operatorname{Ns}(X)$ if and only if both $E$ and $E^{\vee}$ are numerically effective on $X$.

Proof. Let $E$ be in $\mathrm{Ns}(X)$, and $f: C \rightarrow X$ be a morphism of a smooth projective curve. By definition, $f^{*} E$ is semistable of degree 0 . The minimal slope of $f^{*} E$ is the slope of some stable quotient bundle, thus by semistability, it has to be nonnegative. As $E^{\vee}$ is in $\mathrm{Ns}(X)$ as well, we conclude that both $E$ and $E^{\vee}$ are numerically effective. Vice-versa, if $E$ and $E^{\vee}$ are numerically effective, then both $f^{*} E$ and $f^{*} E^{\vee}$ are numerically effective on $C$, thus $E$ is in $\mathrm{Ns}(X)$.

Lemma 2.3. If $E \in \operatorname{Ns}(X)$, then $\operatorname{deg}\left(c_{i}(E) \cdot H^{d-i}\right)=0$ for all $i \geq 1$ and all ample line bundles $H$.

Proof. This is the argument of the proof of [8, Theorem 4.1,Proof]): since $E$ is strongly semistable, by boundedness, there are finitely many such natural numbers $\operatorname{deg}\left(c_{i}\left(F^{*} E\right) \cdot H^{d-i}\right)=p^{i} \cdot \operatorname{deg}\left(c_{i}(E)\right)$, one concludes that they are 0 .

Let $E$ be an essentially finite bundle. Let $\langle E\rangle$ be the full subcategory of $\mathcal{C}^{N}(X)$ spanned by $E$, and set $G(E, x)=\operatorname{Aut}^{\otimes}\left(\langle E\rangle, \omega_{x}\right)$. Then there is an exact sequence $1 \rightarrow G(E, x)^{0} \rightarrow G(E, x) \rightarrow G(E, x)^{\text {et }} \rightarrow 1$ where $G(E, x)^{0}$ is local and $G(E, x)^{\text {et }}$ is étale and is a quotient of $\pi_{1}^{\text {et }}(X, x)$. We denote by $\mathcal{C}^{\text {loc }}(X) \subset \mathcal{C}^{N}(X)$ the full subcategory of essentially finite bundles $E$ which have the property that $G(E, x)^{0}=G(E, x)$. We denote by $\pi^{\text {loc }}(X, x)$ the Tannaka group scheme $\lim _{E, G(E, x)^{0}=G(E, x)} G(E, x)^{0}$. This group has been studied in [2] (where it is denoted by $\pi^{F}(X, x)$ ) and in [11]. It is a quotient $k$-group scheme of $\pi^{N}(X, x)$.

Lemma 2.4. On has the following implications.

(1) If $\pi_{1}^{\mathrm{et}}(X, x)=\{1\}$, and $H^{0}\left(X, \Omega_{X}^{1}\right)=0$, then $\pi_{1}^{N}(X, x)=\{1\}$.

(2) If $\pi_{1}^{N}(X, x)=\{1\}$, then $\pi^{\operatorname{loc}}(X, x)=\{1\}$ and $H^{1}\left(X, \mathcal{O}_{X}\right)=0$.

Proof. We first prove (1). Since $G(E, x)^{\text {et }}$ is a quotient of $\pi_{1}^{\text {et }}(X, x)=\{1\}$, one has $G(E, x)^{0}=G(E, x)$. This implies that there is a $n \in \mathbb{N} \backslash\{0\}$ such that $\left(F^{n}\right)^{*} E$ is trivial. Since $H^{0}\left(X, \Omega_{X}^{1}\right)=0$, there is only one connection on $\left(F^{n}\right)^{*} E$, the trivial one. Thus the connection with flat sections $\left(F^{n-1}\right)^{*} E$ is trivial, thus $\left(F^{n-1}\right)^{*} E$ is trivial. Repeating the argument, we see that $E$ is trivial. This finishes the proof of (1).

We prove (2). The absolute Frobenius map $F: X \rightarrow X$ induces a $p$-linear endomorphism $F^{*}$ on $H^{1}\left(X, \mathcal{O}_{X}\right)$. This induces a decomposition

$$
H^{1}\left(X, \mathcal{O}_{X}\right)=H^{1}\left(X, \mathcal{O}_{X}\right)_{\mathrm{ss}} \oplus H^{1}\left(X, \mathcal{O}_{X}\right)_{\text {nilp }},
$$


where $F^{*}$ is bijective on the first factor and nilpotent on the second factor. Since

$$
H^{1}\left(X, \mathcal{O}_{X}\right)_{\mathrm{ss}}=H^{1}\left(X_{\mathrm{et}}, \mathbb{Z} / p\right) \otimes_{\mathbb{F}_{p}} k=\operatorname{Hom}\left(\pi^{\mathrm{et}}(X), \mathbb{Z} / p\right) \otimes_{\mathbb{F}_{p}} k,
$$

one concludes $H^{1}\left(X, \mathcal{O}_{X}\right)_{\mathrm{ss}}=0$. Let $0 \neq y \in H^{1}\left(X, \mathcal{O}_{X}\right)_{\text {nilp. }}$. There exists $t \in \mathbb{N} \backslash\{0\}$ such that $\left(F^{t-1}\right)^{*}(y) \neq 0$, but $\left(F^{t}\right)^{*}(y)=0$. Then $\left(F^{t-1}\right)^{*}(y)$ defines an $\alpha_{p}$-torsor, a contradiction. This proves $H^{1}\left(X, \mathcal{O}_{X}\right)=0$.

Lemma 2.5. If $\pi^{N}(X, x)=\{1\}$, the line bundles in $\mathrm{Ns}(X)$ are trivial.

Proof. As both $L$ and $L^{\vee}$ are numerically effective on $X, L$ is numerically equivalent to 0 . The group $\operatorname{Num}_{0}(X) / \operatorname{Alg}_{0}(X)$ is a finite group. As $H^{1}\left(X, \mathcal{O}_{X}\right)=0$, the Picard scheme of $X$ is a reduced point. Hence $L$ has finite order. But any nontrivial line bundle of finite order on $X$ defines a torsor over $X$ under a non-trivial finite group-scheme, a contradiction as $\pi^{N}(X, x)$ is trivial. So $L$ is trivial.

Lemma 2.6. (See [8, Theorem 4.1] Let $E \in \mathrm{Ns}(X)$. Then the following properties hold true.

(a) $E$ is semistable with respect to $H$.

(b) If $0 \subset E_{0} \subset E_{1} \subset \ldots \subset E_{n}=E$ is the Jordan-Hölder filtration (or the stable filtration) of $E$, then each subquotient $E_{i} / E_{i-1}$ is locally free, with $\operatorname{deg}\left(c_{j}\left(E_{i} / E_{i-1}\right) \cdot H^{d-i}\right)=0$, for all $j \geq 0$, all $i>0$ and all ample line bundles $H$.

Now we continue with the proof of Theorem [1.2. For $E$ in $\mathrm{Ns}(X)$, we want to show that $E$ is trivial. We may assume, by induction on the rank, that if $W \in \operatorname{Ns}(X)$, with $\operatorname{rank} W<r=\operatorname{rank}(E)$, then $W$ is trivial, using Lemma 2.5. Define a sequence of bundles on $X$ by $E_{0}:=E$, and $E_{n}=F^{*}\left(E_{n-1}\right)$, for $n \geq 1$. This a sequence of bundles on $X$ and $\operatorname{deg}\left(c_{i}\left(E_{n}\right) \cdot H^{d-i}\right)=0$ for all $n \geq 0$ and all $i \geq 1$.

Lemma 2.7. $E_{i} \not E_{j}$, for all $i, j$ with $i \neq j$.

Proof. Assume there are $i, j$ such that $i<j$ and $E_{i} \simeq E_{j}$. Then $\left(F^{t}\right)^{*}\left(E_{i}\right) \simeq E_{i}$, where $t=j-i \neq 0$. If $E_{i}$ is not trivial, by the theorem of Lange-Stuhler [6. Satz 1.4], $E_{i}$ becomes trivial on a non-trivial étale finite covering of $X$. As $\pi^{\text {et }}(X, x)=\{1\}$, one must have $E_{i}$ trivial. But by definition, $E_{i}=\left(F^{i}\right)^{*}\left(E_{0}\right)$, thus $G\left(\left\langle E_{0}\right\rangle, x\right)$ is local, thus $E_{0}$ is trivial as $\pi^{\text {loc }}(X, x)$ is trivial by Lemma 2.4. 2).

Lemma 2.8. In order to prove Theorem 1.2, we may assume that all the $E_{i}$ are stable on $X$

Proof. Let $n \geq 1$ be a natural number. Then $E_{n}=\left(F^{n}\right)^{*}\left(E_{0}\right)$ has to be semistable. Indeed, if there was a subsheaf $W \subset E_{n}$ with $\operatorname{deg}\left(c_{1}(W) \cdot H^{d-1}\right)>0$, then for a smooth complete intersection curve $C=\cap_{1}^{d-1} D_{i}$, with $D_{i}$ in some larger power of the linear system $H,\left.W\right|_{C}$ would be a subsheaf of $\left.E\right|_{C}$ with degree $>0$, a contradiction. 
If $E_{n}$ is trivial, we conclude as in Lemma 2.7 that $E_{0}$ is trivial as well.

Suppose that $E_{n}=\left(F^{n}\right)^{*}\left(E_{0}\right)$ is strictly semistable. For $m \geq n$, let $s(m)$ be the number of stable components of the Jordan-Hölder components of $E_{m}$. It is clear that $s(m)$ is a non-decreasing function of $m$. As the set $\{s(m), m \geq n\}$ is bounded above by $r=\operatorname{rank}(E), s(m)$ is constant for all $m \geq n_{0}$ for some $n_{0} \geq 0$. So if $W_{m} \subset E_{m}$ is the socle of $E_{m}$, then $F^{*} W_{m}=W_{m+1}$ is the socle of $E_{m+1}$ for any $m \geq n_{0}$. The sequence $W_{n}, n \geq n_{0}$ is a sequence of stable subbundles of $E_{n}$. By Lemma 2.6, one has $\operatorname{deg}\left(c_{i}\left(W_{n}\right) \cdot H^{d-i}\right)=0 \forall i \geq 1, \forall n \geq n_{0}$. So by Lemma 2.2, together with [8, Theorem 5.1], we conclude $W_{n} \in \operatorname{Ns}(X) \forall n \geq n_{0}$, hence $E_{n} / W_{n} \in \mathrm{Ns}(X) \forall n \geq n_{0}$ as well.

We apply the induction hypothesis to $\left\{W_{n}, n \geq n_{0}\right\}$ and $\left\{E_{n} / W_{n}, n \geq n_{0}\right\}$, to assert that $\left\{W_{n}, n \geq n_{0}\right\}$ and $\left\{E_{n} / W_{n}, n \geq n_{0}\right\}$ are trivial bundles on $X$. Then $E_{n}$ are extensions of trivial bundles $\forall n \geq n_{0}$. Applying Lemma 2.4 (2), we conclude that $E_{n}$ is trival. But $E_{n} \simeq\left(F^{a}\right)^{*}\left(E_{n-a}\right), 0 \leq a \leq n$. Since $\pi^{N}(X, x)=\{1\}$, $E_{n-a}$ is trivial as well. This finishes the proof.

We continue with the proof of Theorem 1.2. Let $E$ be in $\mathrm{Ns}(X)$. Let $M$ be the moduli space of $\mu$-stable bundles of degree 0 , which is open in the moduli of $\chi$-stable torsionfree sheaves with Hilbert polynomial $p_{E}=p_{\mathcal{O}_{X}}$, as constructed by Langer in [7, Theorem 4.1]. It is a quasi-projective scheme, of finite type over $k$.

Define $T:=\left\{E_{0}, E_{1}, \ldots\right\}$ as a sublocus of $M_{\text {red }}$. Here we identify a $\mu$-stable bundle $W$ with $\mu(W)=0, p_{W}=p_{\mathcal{O}_{X}}$ of rank $r$ with its moduli point $|W| \in M$. Let $N$ be the Zariski closure of $T$ in $M_{\text {red }}$. We give $N$ the reduced structure. By Lemma 2.7, the dimension of $N$ is at least 1.

Consider the decomposition of $N$ into its irreducible components, $A_{i}, i \in I$, and $N_{j}, j \in J$. This labeling is chosen such that $A_{i}$ is finite $\forall i \in I$, and $T \cap N_{j}$ is infinite $\forall j \in J$. Recall that $F: X \rightarrow X$ is the absolute Frobenius morphism of $X$. Let $V: M \cdots \cdots \cdots \rightarrow M$ be the Verschiebung, which is the rational map defined by $[W] \mapsto\left[F^{*} W\right]$. As $F$ preserves $T, V$ maps $N$ into itself rationally. Moreover, since the image of this rational map contains $E_{n}$ for all $n \geq 1, V$ is dominant on the Zariski closure of $\left\{E_{1}, E_{2}, \ldots\right\}$. This implies that $V$ maps each $N_{i}$ into some other $N_{j}$, dominantly. We conclude that $V$ induces a permutation of the set $J$. Hence some nonzero power of $V$ preserves each $N_{j}$.

We can now argue precisely as [3, Section 3]. We choose a scheme $S$, smooth, of finite type, geometrically irreducible over $\mathbb{F}_{q}$, such that

(a) $X$ has a model smooth projective $X_{S} \rightarrow S$,

(b) $M$ has a flat model $M_{S} \rightarrow S$,

(c) all the irreducible components $N_{j}$ of $N$ have a flat model $N_{j S} \rightarrow S$,

(d) $V$ has a model $V_{S}$ on $M_{S}$.

Recall by Langer's theorem [7, Theorem 4.1], $M_{S} \rightarrow S$ universally corepresents the functor of families of stable bundles on the closed fibres of $X_{Y} \rightarrow Y$, thus 
in particular, for all closed points $s \in Y$, one has $M_{S} \times_{S} s=M_{s}$, where $Y$ is any Noetherian scheme over $S$. Applying [3, Corollary 3.11], we obtain that the specialization $V_{S} \times_{S} s$ of $V_{S}$ over closed points $s \in S$ is the Verschiebung of $M_{s}$. We argue as in the proof of [3, Theorem 3.14] to show that Hrushovski's theorem [5. Corollary 1.2] implies the existence of closed points $u$ in each irreducible component $N_{i S}$ of $N_{S}$, mapping to a closed points $s \in S$, such that $V_{s}^{m}$ is defined on $u$ and fulfills $F_{X_{s}}^{m}(u)=u$. Here $F_{X_{s}}: X_{s} \rightarrow X_{s}$ is the absolute Frobenius endomorphism of $X_{s}$. Applying as in loc. cit. Lange-Stuhler Theorem [6, Satz 1.4], we conclude that $N_{j}=\emptyset$ for all $j \in J$.

Thus $T$ has only finitely many components $A_{i}$ of dimension 0 . This implies that $T$ is finite, thus we find $n \in \mathbb{N}$ and $t \in \mathbb{N} \backslash\{0\}$ such that $\left(F^{t}\right)^{*} E_{n} \cong E_{n}$. Applying again Lange-Stuhler Theorem loc. cit., this shows that $E_{n}$ is trivial. This implies by definition that $E_{m}, m \geq n$ is trivial, and by Lemma 2.4 (2) that $E_{m}, 0 \leq m<n$ are trivial as well. This finishes the proof.

Remark 2.9. Over $k=\overline{\mathbb{F}}_{p}$, we do not need Hrushovski's theorem. Indeed, once we know that $N$ is 0 -dimensional, we can apply the theorem of Lange-Stuhler directly.

\section{REMARKS}

3.1. If one knew that the Verschiebung $V: M \cdots \cdots \cdots \rightarrow M$ introduced in the proof of Theorem 1.2 was dominant, then we could apply Hrushovski's theorem directly to it and the proof would be much more direct. In fact, this would prove that torsion points are dense in the sense of Theorem [3, Theorem 3.14]. We do not know this in general, but do know it in dimension 1 [15, Theorem 6].

3.2. Let $X$ be a smooth projective variety defined over a field $k$. We know that if $k$ is finite, $E$ lies in $\mathrm{Ns}(X)$ if and only $E$ lies in $\left.\mathcal{C}^{N} X\right)$, that is is essentially finite. So one may be tempted to argue that of a model $E_{S}$ of $E \in \mathrm{Ns}(X)$ over $X_{S} \rightarrow S$ has the property that over a dense set of closed points $s \in S$, $E \otimes k(s) \in \mathcal{C}^{N}\left(X \times{ }_{S} s\right)$. But this is not true ([12]). We are grateful to Holger Brenner for pointing out this reference to us.

3.3. In [14, Chapter II,Proposition 8], Nori shows that if $X$ is projective smooth and geometrically irreducible, then $\pi_{1}^{N}(X, x)$ is a birational invariant among the smooth projective models of $k(X)$. Langer in [8, Lemma 8.3] shows that blow ups with smooth centers do not affect $\pi^{S}(X, s)$ and raises the question whether Nori's result extends to $\pi^{S}(X, x)$. Theorem 1.2 shows that this is true under the assumption that $\pi^{N}(X, x)$ is trivial.

\section{REFERENCES}

[1] Biswas, I., Parameswaran A.J.,Subramanian S. : Monodromy Group For A Strongly Semistable Principal Bundle Over A Curve, Duke Mathematical Journal, 132 no1 (2006). 
[2] Esnault, H, Hai P.-H., Sun, X.: On Nori's Fundamental Group Scheme, Progress in Mathematics, Vol. 265 (2007), 377-398.

[3] Esnault, H., Mehta, V.: Simply Connected manifolds in Characteristic $p>0$ have no nontrivial stratified bundles, arxiv/0907.3375.

[4] Gieseker, D.: Stable vector bundles and the Frobenius morphism, Ann. Sc. Éc. Norm. Sup. 4-ième série, tome 6, no1 (1973), 95-101.

[5] Hrushovski, E.: The Elementary Theory of Frobenius Automorphisms, arxiv/0406514v1.

[6] Lange, H., Stuhler, U .: Vektorbündel auf Kurven und Darstellungen der algebraischen Fundamentalgruppe, Math. Zeit. 156 (1977), 73-83.

[7] Langer, A.: Moduli spaces of sheaves in mixed characteristics, Duke math. J. 124 no3 (2004), 571-586.

[8] Langer, A.: On the S-Fundamental group scheme, arXiv/0905.4600.

[9] Langer, A.: On the S-Fundamental group scheme II, preprint 2009.

[10] Malcev, A.: On isomorphic matrix representations of infinite groups, Rec. Math. [Mat. Sbornik] N.S. 8 (50) (1940), 405-422.

[11] Mehta, V., Subramanian, S.: Some remarks on the local fundamental group scheme, Proc. Ind. Acad. of Sc. Math. Sci. 118, no.2 (2008), 207-211.

[12] Monsky, P.: Hilbert-Kunz functions in a family: line $S_{4}$-quartics, J. of Algebra 208 (1998), no1, 249-371.

[13] Narasimhan, M.S., Seshadri, C.S.: Stable bundles and unitary bundles on a compact Riemann surface, Proc. Nat. Acad. Sci. U.S.A. 52 (1964), 207- 211.

[14] Nori, M : The Fundamental Group-Scheme, Proc. Ind. Acad. Sc. Math. Sci., 91 no.2 (1982), $73-122$

[15] Osserman, B.: The generalized Verschiebung map for curves of genus 2, Math. Ann. 336 (2006), 963-986.

Universität Duisburg-Essen, Mathematik, 45117 Essen, Germany

E-mail address: esnault@uni-due.de

Mathematics, Tata Institute, Homi Bhabha Road, Mumbai 400005, India

E-mail address: vikram@math.tifr.res.in 